\title{
Placing Two Disks in a Convex Polygon
}

\author{
Sung Kwon Kim* $\quad$ Chan-Su Shin ${ }^{\dagger} \quad$ Tae-Cheon Yang ${ }^{\ddagger}$
}

Keywords: Computational geometry, optimization, convex polygon.

\section{Introduction}

Let $P$ be a convex polygon of $n$ vertices. We consider a problem (and some of its variants) of finding a pair of largest equiradial non-overlapping disks in $P$. Regarding $P$ as a sheet of paper, one can fold $P$ along the bisector of the line segment connecting the centers of the disks. Then those two disks coincide each other in the folded polygon. This means the problem is identical to that of finding a largest disk which can be hidden with $P$ by one straight fold. Both sides of the hidden disk should be covered by $P$. For an illustration, refer to Figure 1 (a). This problem is well motivated from the gift wrapping problem that determine whether a gift is wrapped up (or hidden) using a given paper through one-straight fold.

Consider a line $l$ passing through two points on two different edges of $P$. The line $l$ divides $P$ into two convex subpolygons $P_{1}(l)$ and $P_{2}(l)$. We want to find a folding line $l$ which gives two largest equiradial non-overlapping disks, one in $P_{1}(l)$ and the other in $P_{2}(l)$. If we fold $P$ along $l$, then the disks coincide in the folded polygon. A disk of the same radius can be hidden in $P$. We then define two problems according to the type of the folding line as follows:

P1: Find an optimal folding line for a convex polygon $P$. Note that a folding line in this problem does not necessarily pass through vertices of $P$.

P2: Find an optimal folding line for a convex polygon $P$ so that it passes through two vertices of $P$.

Problem P1 was investigated in [4, 3]. Biedl et al. [4] considered the problem for a simple polygon, and presented a polynomial-time algorithm; for a convex polygon, their algorithm runs in $O\left(n^{2}\right)$ time. Recently, Bespamyatnikh [3] reduced the time bound to $O\left(n \log ^{3} n\right)$ by using parametric search technique [7]. In this paper, we present an algorithm for a convex polygon whose running time is $O(n \log n)$. Our algorithm does not employ parametric search technique which is known to be hard to implement [1].

For problem P2, to our knowledge, no algorithm has been known so far. We solve P2 in $O\left(n^{2} \log ^{2} n\right)$ time by adapting parametric search technique.

\footnotetext{
${ }^{*}$ Dept. of Computer Science and Engineering, Chung-Ang Univ., Korea. skkim@cau.ac.kr

$\dagger$ Dept. of Computer Science, HKUST, Hong Kong. cssin@cs.ust.hk

$\ddagger$ School of Information Science, Kyungsung Univ., Korea. tcyang@csd.kyungsung.ac.kr
} 
Let us now modify these problems in a slightly different way. As above, define two subpolygons $P_{1}(l)$ and $P_{2}(l)$ of $P$ by a line $l$ intersecting $P$. Let $r_{i}(l)$ be the radius of the largest disk inscribed in $P_{i}(l)$ for $i=1,2$. Our new problems are to find a separating line $l$ that gives

$$
\max _{l}\left(\min \left(r_{1}(l), r_{2}(l)\right)\right) .
$$

Two different problems are possible according to the type of a separating line:

Q1: Find an optimal separating line when the maximum in the above equation is taken over all lines intersecting $P$.

Q2: Find an optimal separating line when the maximum in the above equation is taken over all lines passing through two vertices of $P$.

As will be seen later, problem $\mathbf{Q 1}$ is identical to problem $\mathbf{P 1}$, so we can solve $\mathbf{Q 1}$ in $O(n \log n)$ time. Hence, the problem of finding two largest non-overlapping disks in a convex polygon is equivalent to problems $\mathbf{P 1}$ and Q1. But problem Q2 is entirely different from problem P2. We will prove that $\mathbf{Q 2}$ can be solved in $O\left(n \log ^{2} n\right)$ time by a divide-and-conquer approach.

Besides, there is a related problem of finding two largest disks whose union covers (the boundary of) a convex polygon, instead of inscribing two disks in a convex polygon. The problem was investigated, together with some variants, in [9].

Throughout this paper, we denote by $P$ a convex polygon of $n$ vertices. As the general-position assumption in other geometric algorithms, we assume here that no four or more edges of $P$ touch a common disk. Here, "touch" means just "contact" (not pierce). In addition, we assume that there are no parallel edges in $P$. These assumptions about input polygons can be easily removed by several techniques [5].

\section{Algorithm for problem P1}

In this section, we will consider the problem $\mathbf{P 1}$, which is to find a largest disk hidden in $P$ by one straight fold. As stated in the previous section, this is equivalent to finding two largest equiradial non-overlapping disks in $P$, so in what follows, we will explain how to find those disks in $P$.

Let $D_{1}$ and $D_{2}$ be two optimal equiradial disks in $P$ and let $r^{\star}$ be their radius. We shall denote the boundary of some closed region $R$ by $\partial R$. Let us begin with stating two simple, but crucial, lemmas.

Lemma $1 D_{1}$ and $D_{2}$ touch each other; and each of $D_{1}$ and $D_{2}$ touches (at least) two edges of $P$.

Proof: If $D_{1}$ and $D_{2}$ do not touch each other, we can immediately enlarge both disks to obtain a pair of larger disks. So, $D_{1}$ and $D_{2}$ must touch each other. If $D_{1}$, touching $D_{2}$, touches none of the edges of $P$, then $D_{1}$ can be moved away from $D_{2}$ so that they no longer touch. If $D_{1}$, touching $D_{2}$, touches only one edge of $P$, then $D_{1}$ can 


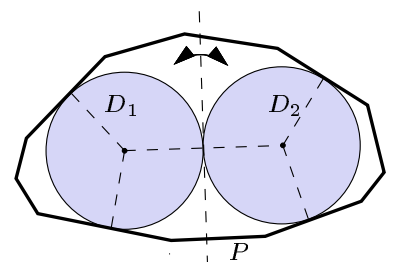

(a)

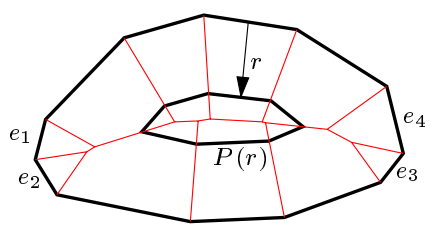

(b)

Figure 1: (a) A configuration of two optimal disks $D_{1}$ and $D_{2}$. (b) A shrunken polygon $P(r)$ of $P$. The thin lines represent the medial axis of $P$. Note that some edges of $P$ such as $e_{1}, e_{2}, e_{3}, e_{4}$ disappear in $P(r)$.

still be moved so that it no longer touches $D_{2}$.

From the above lemma, $D_{i}$ has (at least) three touching points: (at least) two edge-touching points and one disk-touching point. Note that $D_{i}$ may have (at most) three edge-touching points by the general-position assumption. However, assume, for a simplicity, that $D_{i}$ touches exactly two edges and the other disk (refer to Figure 1 (a)). Since such three touching points fix each disk immovable in $P$, they must form an acute-triangle of all inner angles $<\pi / 2$.

Lemma 2 Two edge-touching points and one disk-touching point of $D_{i}$ form an acute-triangle containing the center of $D_{i}$ for $i=1,2$.

Let us now describe an $O(n \log n)$-time algorithm for problem P1. The locations at which the centers of $D_{i}$ 's may lie are closely related to the medial axis [8] of $P$. The medial axis of $P$ is defined as the locus of all centers of disks contained in $P$ that touch $\partial P$, at two or three points (see Figure 1 (b)). In other words, the medial axis of $P$ is a skeleton of the Voronoi diagram for the edges of $P$, excluding their end vertices. Thus, the medial axis consists of Voronoi vertices and Voronoi edges (which are straight line segments). It can be computed in linear time [2].

From Lemma 1, we know that the centers of $D_{i}$ 's lie on some edges of the medial axis of $P$. A straightforward way to solve $\mathbf{P 1}$ is to compute two largest equiradial disks decided by each pair of edges of the medial axis of $P$. The maximum among the computed radii is what we want. It takes $\Omega\left(n^{2}\right)$ time in total. As will be seen later, we do not need to consider all such $O\left(n^{2}\right)$ pairs; only $O(n)$ pairs are enough.

Before explaining it, we will first consider the decision version of problem $\mathbf{P 1}$ as follows: Given a fixed radius $r>0$, decide whether two non-overlapping disks of radius $r$ can be placed in $P$. Let $P(r)$ be a shrunken polygon of $P$ that is a locus of points in $P$ at distance $r$ from $\partial P$ (see Figure 1 (b)). If a disk of radius $r$ has its center on any location of $\partial P(r)$, then the disk is entirely contained in $P$. The shrunken polygon $P(r)$ can be easily constructed in linear time by walking around $\partial P[6,10]$. Note that some edges of $P$ may disappear in $\partial P(r)$. Next, compute a farthest vertex pair (i.e., diameter pair) of $P(r)$ in linear time [8]. If the diameter is greater than or equal to $2 r$, then we can place two non-overlapping disks of radius $r$ 
with their centers at the farthest vertex pair of $P(r)$. Otherwise, we have no feasible placements.

Lemma 3 Given a fixed radius $r>0$, one can decide in linear time whether two non-overlapping disks of radius $r$ can be placed in $P$, i.e., whether $r \leq r^{\star}$, where $r^{\star}$ is the radius of two largest equiradial non-overlapping disks in $P$.

Let $M$ be the medial axis of $P$. Consider a vertex $\nu$ of $M$. Then we can draw a disk of some radius $r$, centered at $\nu$ and touching three edges of $P$ that define $\nu$ in $M$. Collect all such radii associated with the vertices of $M$, and sort them in non-decreasing order, $r_{1}, r_{2}, \cdots, r_{m}$. Note that $m=O(n)$, and the disk of radius $r_{m}$ is the largest disk inscribed in $P$. Clearly, there is an index $j(1 \leq j<m)$ such that $r_{j} \leq r^{\star}<r_{j+1}$. We can find $j$ in $O(n \log n)$ time by performing a binary search on the sorted list of $r_{i}$ 's with the help of our decision algorithm (see Lemma 3).

Shrink $P$ by $r_{j}$ to get $P\left(r_{j}\right)$. Some edges of $P$ may disappear in $\partial P\left(r_{j}\right)$. Expand $P\left(r_{j}\right)$ by $r_{j}$ outwardly and denote by $P^{\prime}$ the expanded polygon. Refer to Figure 2 (b). Since $P^{\prime}$ is obtained by simply scaling $P\left(r_{j}\right)$ by $r_{j}$, every edge of $P\left(r_{j}\right)$ appears in $\partial P^{\prime}$. However, some edges of $P$ may not appear in $\partial P^{\prime}$, and thus $P \subseteq P^{\prime}$.

Lemma 4 Let $r^{\prime}$ be the radius of two largest non-overlapping disks in $P^{\prime}$. Then, $r^{\prime}=r^{\star}$.

Proof: It clearly holds that $r^{\star} \leq r^{\prime}$ because $P \subseteq P^{\prime}$. We assume that $r^{\star}<r^{\prime}$, and will show that this leads to a contradiction.

Let $P\left(r^{\prime}\right)$ and $P^{\prime}\left(r^{\prime}\right)$ be the shrunken polygons of $P$ and $P^{\prime}$ by $r^{\prime}$, respectively. We show that $P\left(r^{\prime}\right)$ is actually identical to $P^{\prime}\left(r^{\prime}\right)$, i.e., $P\left(r^{\prime}\right)=P^{\prime}\left(r^{\prime}\right)$. If they are identical, then we can place two disks of radius $r^{\prime}$ in $P$, which contradicts to the optimality of $r^{\star}$ for $P$. Thus, we can conclude that $r^{\star}=r^{\prime}$.

Since $P\left(r^{\prime}\right) \subseteq P^{\prime}\left(r^{\prime}\right)$ clearly, we assume that $P\left(r^{\prime}\right) \subset P^{\prime}\left(r^{\prime}\right)$. This implies there must be an edge $e_{1}$ of $P\left(r^{\prime}\right)$ such that no edges of $P^{\prime}\left(r^{\prime}\right)$ contain $e_{1}$ entirely. Let $e$ be the original edge of $e_{1}$ in $P$, that is, $e$ is shrunken to $e_{1}$ in $P\left(r^{\prime}\right)$. Let $e_{2}$ be the shrunken edge of $e$ in $P\left(r_{j}\right)$. In fact, $e_{2}$ does not exist. If $e_{2}$ exists in $P\left(r_{j}\right)$, then $e_{2}$ would be expanded to some edge $e_{3}$ of $P^{\prime}$. Remind that $P^{\prime}$ is an expanded polygon of $P\left(r_{j}\right)$ by $r_{j}$, and thus $e \subseteq e_{3}$. The edge $e_{3}$ is again shrunken to an edge $e_{4}$ in $P^{\prime}\left(r^{\prime}\right)$. Finally, $e_{1} \subseteq e_{4}$, which contradicts to the fact that no edges in $P^{\prime}\left(r^{\prime}\right)$ contain entirely $e_{1}$. Thus $e_{2}$ does not exist in $P\left(r_{j}\right)$. Note that $e$ is shrunken to an empty edge in $P\left(r_{j}\right)$, while, to (a non-empty edge) $e_{1}$ in $P\left(r^{\prime}\right)$ for $r^{\prime}>r_{j}$. This is impossible, so $P\left(r^{\prime}\right)=P^{\prime}\left(r^{\prime}\right)$.

Lemma 4 means that it is enough to compute two largest disks in $P^{\prime}$, instead in $P$. In what follows, we will show $P^{\prime}$ has a nice property to compute the disks and present in detail how to compute them. Consider two shrunken polygons $P\left(r_{j}\right)$ and $P\left(r_{j+1}\right)$. Let $M^{\prime}$ be the part of $M$ belonging to $P\left(r_{j}\right) \backslash P\left(r_{j+1}\right)$, that is, the clipped part of $M$ by $P\left(r_{j}\right) \backslash P\left(r_{j+1}\right)$ (see Figure $2(\mathrm{a})$ ). Note that the region $P\left(r_{j}\right) \backslash P\left(r_{j+1}\right)$ includes $\partial P\left(r_{j}\right)$, but does not include $\partial P\left(r_{j+1}\right)$. Since $r_{j}$ is the maximum among all 


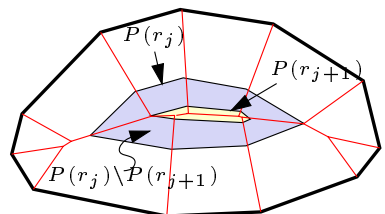

(a)

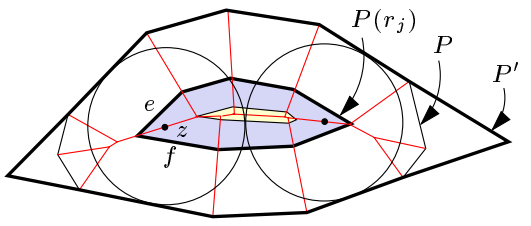

(b)

Figure 2: (a) A shaded region represents $P\left(r_{j}\right) \backslash P\left(r_{j+1}\right)$. (b) A convex polygon $P$, a shrunken polygon $P\left(r_{j}\right)$, and an expanded polygon $P^{\prime}$.

$r_{i}$ 's $\leq r^{\star}$, there are no vertices of $M$ inside $P\left(r_{j}\right) \backslash P\left(r_{j+1}\right)$ (possibly, some vertices of $M$ may be on $\left.\partial P\left(r_{j}\right)\right)$. In other words, $M^{\prime}$ consists of only pieces of some edges of $M$ clipped by $P\left(r_{j}\right) \backslash P\left(r_{j+1}\right)$. We want to notice here that $M^{\prime}$ is actually a subset of a medial axis for $P\left(r_{j}\right)$. Since $r_{j} \leq r^{\star}<r_{j+1}$, the optimal disks, $D_{1}$ and $D_{2}$, for $P^{\prime}$ have their centers on some edges of $M^{\prime}$. Suppose that one of the disks, say $D_{1}$, has its center on an edge $z$ of $M^{\prime}$. As shown in Figure $2(\mathrm{~b})$, since $z$ is a medial axis edge for $P\left(r_{j}\right)$, it is defined by two edges $e, f$ of $\partial P\left(r_{j}\right)$. The edges $e$ and $f$ have to be adjacent on $\partial P\left(r_{j}\right)$. Remind that $P^{\prime}$ is an expanded polygon of $P\left(r_{j}\right)$ by $r_{j}$. So, the expanded edges of $e$ and $f$ are also adjacent on $\partial P^{\prime}$. From this observation, we have the following lemma.

Lemma 5 For each optimal disk for $P^{\prime}$, two edges of $\partial P^{\prime}$ at which the disk touches are adjacent on $\partial P^{\prime}$.

The remaining is to compute, for each pair of vertices of $P^{\prime}$, two largest disks determined by its incident four edges. For one vertex pair, we can easily compute the optimal disks in $O(1)$ time [4], so we can consider all possible $O\left(n^{2}\right)$ pairs in $O\left(n^{2}\right)$ time. But, the following lemma states it is sufficient to consider only $O(n)$ antipodal pairs of vertices of $P^{\prime}$. A pair of vertices is said to be antipodal if it admits parallel supporting lines [8]. All antipodal pairs of a convex polygon are found in linear time [8].

Lemma 6 To compute two largest disks for $P^{\prime}$, we need consider only $O(n)$ antipodal pairs of vertices of $P^{\prime}$.

Proof: Consider a line $l$ that connects two centers of the optimal disks $D_{1}$ and $D_{2}$ for $P^{\prime}$. The line $l$ passes through the point at which $D_{1}$ and $D_{2}$ touch each other. By Lemma 2, two edge-touching points of each $D_{i}$ must be located in the opposite sides with respect to $l$. This means one can draw two parallel lines supporting a pair of vertices that are incident to touching-edges; such parallel lines will be perpendicular to $l$.

The above lemma allows us to finally compute two largest disks for $P$ by checking only $O(n)$ antipodal pairs of vertices of $P^{\prime}$ in $O(n)$ time. Since we need $O(n \log n)$ time to sort radii $r_{i}$ 's and find $r_{j}$, our algorithm runs in $O(n \log n)$ time in total. 
Theorem 1 Given a convex polygon $P$ of $n$ vertices, we can find a largest disk in $O(n \log n)$ time that can be hidden in $P$ when $P$ is folded along a line intersecting $P$.

\section{Algorithm for problem P2}

Let us now consider problem P2 of finding a largest disk that can be hidden in a convex polygon $P$ when we fold $P$ along a line passing through two vertices of $P$. This problem is solved in $O\left(n^{2} \log ^{2} n\right)$ by simply adapting a parametric search technique [7].

Parametric search is an optimization technique which can be applied in situations where we seek a maximum parameter $r^{\star}$ satisfying the monotone condition that is met by all $r \leq r^{\star}$ but not by any $r>r^{\star}$. The strategy of the parametric search is to design efficient sequential and parallel algorithms for the corresponding decision problem: decide whether a given parameter $r$ is smaller than or larger than or equal to the maximum parameter $r^{\star}$.

Problem P2 clearly satisfies the monotone condition that one can hide a disk of all radius $r \leq r^{\star}$ in $P$, but not for any radius $r>r^{\star}$. The decision problem for $\mathbf{P 2}$ is as follows: Given a radius $r>0$, decide whether a disk of radius $r$ can be hidden in $P$ when one folds $P$ along a line passing through two vertices of $P$. Refer to Figure 3 (a).

Assume that we have a sequential algorithm $A_{s}$ which runs in $O\left(T_{s}\right)$ time, and a parallel algorithm $A_{p}$ which runs in $O\left(T_{p}\right)$ time using $W_{p}$ processors. Then the optimization problem is solved in $O\left(W_{p} T_{p}+T_{s} T_{p} \log W_{p}\right)$ time; see [7] for details. In this section, we will present a sequential algorithm with $T_{s}=O\left(n^{2}\right)$ and a parallel algorithm with $T_{p}=O(\log n)$ and $W_{p}=O\left(n^{2}\right)$, so $\mathbf{P 2}$ is solved in $O\left(n^{2} \log ^{2} n\right)$ time.

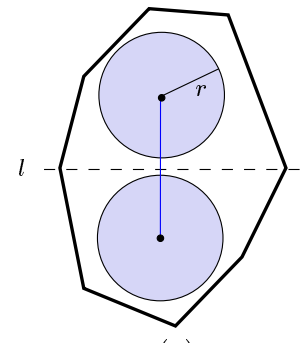

(a)

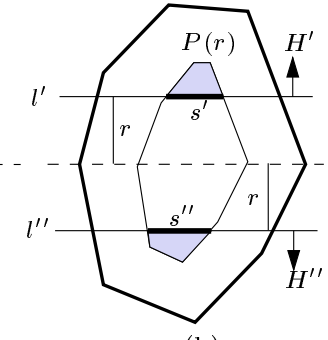

(b)

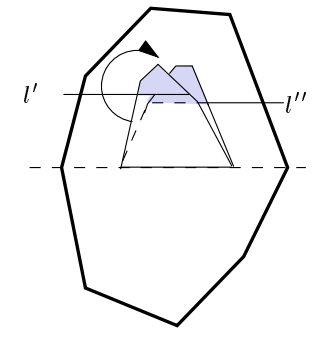

(c)

Figure 3: (a) Two disks of radius $r$ are contained in $P$, i.e., the answer of the decision problem is "yes". (b) Shaded regions are $A^{\prime}$ and $A^{\prime \prime}$. (c) Folding $P(r)$ along $l$.

Let $l$ be a line through two vertices of $P$. For simplicity, regard $l$ as the $x$-axis. Given a value of $r>0$, we will check whether a disk of radius $r$ can be hidden when $P$ is folded along $l$. Let $l^{\prime}$ and $l^{\prime \prime}$ be the lines apart from $l$ by distance $r$ in $+y$ and $-y$ directions, respectively. Let $H^{\prime}$ (resp., $H^{\prime \prime}$ ) be the halfplane bounded below by $l^{\prime}$ (resp., above by $l^{\prime \prime}$ ). See Figure 3 (b). Consider a shrunken polygon $P(r)$ of $P$. 
Define $A^{\prime}=P(r) \cap H^{\prime}$ and $A^{\prime \prime}=P(r) \cap H^{\prime \prime} . A^{\prime}$ (resp., $A^{\prime \prime}$ ) is the region of points at which a disk of radius $r$ can center so that the disk is contained in the upper (resp., lower) subpolygon of $P$ divided by $l$. We fold $P(r)$ along $l$ as shown in Figure 3 (c). In the folded polygon, if $A^{\prime} \cap A^{\prime \prime} \neq \emptyset$, then we can hide a disk of radius $r$ in $P$ by putting its center at a point in $A^{\prime} \cap A^{\prime \prime}$. Otherwise, no disk of radius $r$ can be hidden in $P$.

Hence, we need to check whether $A^{\prime} \cap A^{\prime \prime}$ in the folded polygon of $P(r)$ is empty. One simple way is to compute their actual intersection. However this needs time at least in linear to the number of vertices involved, possibly $\Omega(n)$. Define two line segments, $s^{\prime}=P(r) \cap l^{\prime}$ and $s^{\prime \prime}=P(r) \cap l^{\prime \prime}$. Then the following simple lemma allows us to reduce the checking time down to $O(1)$.

Lemma 7 In the folded polygon of $P(r), A^{\prime} \cap A^{\prime \prime} \neq \emptyset$ if and only if $s^{\prime} \cap s^{\prime \prime} \neq \emptyset$.

Proof: If $s^{\prime} \cap s^{\prime \prime} \neq \emptyset$, then clearly $A^{\prime} \cap A^{\prime \prime} \neq \emptyset$. For a contradiction, assume that $s^{\prime} \cap s^{\prime \prime}=\emptyset$, but $A^{\prime} \cap A^{\prime \prime} \neq \emptyset$. Let $q$ be a point in $A^{\prime} \cap A^{\prime \prime}$. In $P(r), q$ defines two "twin" points, one in $A^{\prime}$ and the other in $A^{\prime \prime}$. Let $\lambda$ be the vertical line segment connecting these twin points. Since $P(r)$ is convex, $\lambda \in P(r)$. So, in $P(r), \lambda$ intersects $s^{\prime}$ and $s^{\prime \prime}$ at $t^{\prime}$ and $t^{\prime \prime}$, respectively. Points $t^{\prime}$ and $t^{\prime \prime}$ are twin points as they coincide at a point, $t$, in the folded polygon of $P(r)$. This implies $t \in s^{\prime} \cap s^{\prime \prime}$, which is a contradiction.

Let us summarize our decision algorithm. First, compute the shrunken polygon $P(r)$. For each vertex $v$ of $P$, do the following steps. (i) For each line $l$ passing through $v$ and $v$ 's non-adjacent vertices of $P$, compute $s^{\prime}$ and $s^{\prime \prime}$ and check if they intersect when folding $P(r)$ along $l$. (ii) If there is a pair of vertices such that $s^{\prime}$ and $s^{\prime \prime}$ intersect, then the answer is "yes". If there is no such pair, the answer is "no".

Let us analyze the time complexity of a sequential implementation of the algorithm. Clearly, the total running time depends on the step (i). For a fixed vertex $v$, we can compute all $s^{\prime}$ and $s^{\prime \prime}$ in linear time by traversing edges of $P(r)$ one by one like the method [8] of finding antipodal pairs of vertices in a convex polygon. This is possible because both $P$ and $P(r)$ are convex. Since we have to perform step (i) for all $n$ vertices of $P$, the total time becomes $O\left(n^{2}\right)$, i.e., $T_{s}=O\left(n^{2}\right)$.

A parallel implementation is more straightforward. Computing $P(r)$ is done in $O(\log n)$ time with $O(n)$ processors as follows: Assign a processor to each medial-axis edge and compute a point on it in constant time whose distance to $\partial P$ is exactly $r$. Next, angular-sort the points with respect to the center of the largest inscribing circle of $P$ in $O(\log n)$ time with $O(n)$ processors. To obtain $P(r)$, just connect the points in the sorted order. For the remaining step, assign a processor to each pair of vertices of $P$ and compute their corresponding $s^{\prime}$ and $s^{\prime \prime}$ in $O(\log n)$ time through a binary search on $\partial P(r)$. As a result, $T_{p}=O(\log n)$ and $W_{p}=O\left(n^{2}\right)$. Plugging these bounds into the parametric search engine, we have the following result.

Theorem 2 Given a convex polygon $P$ of $n$ vertices, we can find a largest disk in $O\left(n^{2} \log ^{2} n\right)$ time that can be hidden in $P$ when $P$ is folded along a line passing through two vertices of $P$. 


\section{Algorithms for problems Q1 and Q2}

In this section, we will consider problems $\mathbf{Q 1}$ and $\mathbf{Q 2}$. Let $r_{i}(l)$ be the radius of the largest disk inscribed in $P_{i}(l)$, where $P_{i}(l)$ for $i=1,2$ is a subpolygon of $P$ divided by a separating line $l$ intersecting $P$.

Problem Q1 is to find a separating line that maximizes the value $\min \left(r_{1}(l), r_{2}(l)\right)$ for all lines $l$ intersecting $P$. We first show that problem Q1 is actually identical to problem P1. An obvious fact is that the optimal two disks of problem Q1 must be equiradial; otherwise, one can shrink the larger one and enlarge the smaller one to increase the value of $\min \left(r_{1}(l), r_{2}(l)\right)$. Another fact is that the two optimal disks touch each other on the separating line. Its proof is quite similar to that of Lemma 1 . These facts directly lead that an optimal separating line in Q1 is just the perpendicular bisector of the line segment connecting the centers of optimal two disks in P1. As a result, problem $\mathbf{Q 1}$ can be solved in $O(n \log n)$ time by Theorem 1. In what follows, we will consider only problem Q2.

Problem Q2 is to find a separating line that maximizes the value $\min \left(r_{1}(l), r_{2}(l)\right)$ for all lines $l$ passing through two vertices of $P$. To solve it, we shall apply a divideand-conquer technique. Fix a vertex $v$ of $P$ and number the vertices of $P$ counterclockwise $v_{0}(=v), v_{1}, \cdots, v_{n-1}$. Denote by $l_{j}$ a line connecting $v$ and (its non-adjacent vertex) $v_{j}$ for $2 \leq j \leq n-2$. Then $l_{j}$ partitions $P$ into two subpolygons $P_{1}\left(l_{j}\right)$ and $P_{2}\left(l_{j}\right) ; P_{1}\left(l_{j}\right)$ consists of vertices $v_{0}, v_{1}, \cdots, v_{j}$ and $P_{2}\left(l_{j}\right)$ does of $v_{j}, v_{j+1}, \cdots v_{n-1}, v_{0}$. As the index $j$ increases, $r_{1}\left(l_{j}\right)$ monotonically increases and $r_{2}\left(l_{j}\right)$ monotonically decreases. From this monotonicity, we can easily prove that there are at most two local maxima among the values of $\min \left(r_{1}\left(l_{j}\right), r_{2}\left(l_{j}\right)\right)$ 's, and moreover, they must be consecutive. For simplicity of the algorithm description, assume that we have only one maxima at the line $l_{k}$ for some $2 \leq k \leq n-2$. (For the case that two local maxima exist, a similar argument is used.)

Lemma 8 Let $l$ be a line passing through any pair of vertices $u$ and $w$, where $u, w \in$ $\left\{v_{1}, v_{2}, \cdots, v_{k-1}\right\}$ or $u, w \in\left\{v_{k+1}, v_{k+2}, \cdots, v_{n-1}\right\}$. Then

$$
\min \left(r_{1}(l), r_{2}(l)\right)<\min \left(r_{1}\left(l_{k}\right), r_{2}\left(l_{k}\right)\right)
$$

Proof: Assume that $u=v_{i}$ and $w=v_{j}$, where $1 \leq i<j \leq k-1$. Then the counterclockwise ordering on $\partial P$ is $v_{0}=v, v_{i}, v_{j}$, and $v_{k}$. For a contradiction, suppose that the inequality does not hold for the pair $\left(v_{i}, v_{j}\right)$. Consider a line $l_{j}$ through $v$ and $v_{j}$. Then, from the monotonicity, $\min \left(r_{1}\left(l_{j}\right), r_{2}\left(l_{j}\right)\right)$ is strictly larger than $\min \left(r_{1}\left(l_{k}\right), r_{2}\left(l_{k}\right)\right)$. This means the line $l_{j}$ is a new maximum for $v$, which contradicts to the optimality of $l_{k}$.

From this lemma, we need to consider only pairs $(u, w)$ of vertices with $u \in$ $\left\{v_{0}, v_{1}, \cdots, v_{k}\right\}$ and $w \in\left\{v_{k}, v_{k+1}, \cdots, v_{n-1}, v_{0}\right\}$. Consequently, the number of pairs to be considered is reduced by almost half. We now apply a divide-and-conquer technique with two vertex chains, $S_{1}=\left\{v_{0}, v_{1}, \cdots, v_{k}\right\}$ and $S_{2}=\left\{v_{k}, \cdots, v_{n-1}, v_{0}\right\}$. 
The recursive part is summarized as follows: (i) Pick the middle vertex, $k / 2$-th one, from $S_{1}$ and denote it by $v$. (ii) Find a vertex $v^{\prime}$ in $S_{2}$ that gives the local maximum for $v$. Note that $l_{k}$ is a line passing through $v$ and $v^{\prime}$. Then $v$ divides $S_{1}$ into two chains of equal size, $S_{11}$ and $S_{12}$, in the counterclockwise order. Also, $v^{\prime}$ divides $S_{2}$ into two chains, $S_{21}$ and $S_{22}$, in the counterclockwise order. By Lemma 8 , we need to consider only vertex-pairs between $S_{11}$ and $S_{21}$, and between $S_{12}$ and $S_{22}$. As a result, we further eliminate a half of vertex-pairs. (iii) Perform steps (i)-(ii) recursively with $S_{1}=S_{11}$ and $S_{2}=S_{21}$, and with $S_{1}=S_{12}$ and $S_{2}=S_{22}$. Recursion stops when $S_{1}$ consists of only one vertex; at that time, the local maximum for the vertex is found in $S_{2}$ as in (ii).

Let us analyze time complexity. Since a largest inscribed disk of a convex polygon can be found in linear time, the value of $\min \left(r_{1}(l), r_{2}(l)\right)$ can be computed in linear time by finding largest disks in $P_{1}(l)$ and $P_{2}(l)$. The values of $\min \left(r_{1}\left(l_{j}\right), r_{2}\left(l_{j}\right)\right)$ have at most two (consecutive) local maxima, so we can perform a binary search to locate $l_{k}$. Step (ii) thus takes $O(n \log n)$ time. The recursion will stop after $O(\log n)$ times of recursive calls. Hence, the total running time is $O\left(n \log ^{2} n\right)$.

Theorem 3 Given a convex polygon $P$ of $n$ vertices, we can find the maximum value of the minimum radius of two disks of $P$ in $O\left(n \log ^{2} n\right)$ time if the separating lines are restricted to ones through two vertices of $P$.

\section{References}

[1] P.K. Agarwal and M. Sharir. Efficient algorithms for geometric optimization. Tech. Report CS-1996-19, Dept. Computer Science, Duke University, 1996.

[2] A. Aggarwal, L.J. Guibas, J. Saxe, and P.W. Shor. A linear-time algorithm for computing the Voronoi diagram of a convex polygon. Discrete Comput. Geom., 4(6):591-604, 1989.

[3] S. Bespamyatnikh. Efficient algorithm for finding two larget empty circles. In Proc. European Workshop on Computational Geometry, page to appear, 1999.

[4] T.C. Biedl, E.D. Demaine, M.L. Demaine, A. Lubiw, and G.T. Toussaint. Hiding disks in folded polygons. In Proc. 10th Canad. Conf. Comput. Geom., 1998.

[5] H. Edelsbrunner. Algorithms in Combinatorial Geometry, volume 10 of EATCS Monographs on Theoretical Computer Science. Springer-Verlag, Heidelberg, West Germany, 1987.

[6] M. Held. On the Computational Geometry of Pocket Machining, volume 500 of Lecture Notes Comput. Sci. Springer-Verlag, June 1991.

[7] N. Megiddo. Applying parallel computation algorithms in the design of serial algorithms. J. ACM, 30(4):852-865, 1983.

[8] F.P. Preparata and M.I. Shamos. Computational Geometry: An Introduction. SpringerVerlag, 3rd edition, October 1990.

[9] C.-S. Shin, J.-H. Kim, S. K. Kim, and K.-Y. Chwa. Two-center problems for a convex polygon. In Proc. European Symp. on Algorithm, volume 1461 of Lecture Notes Comput. Sci., pages 199-210. Springer-Verlag, 1998.

[10] T.-C. Yang, S.Y. Shin, and K.-Y. Chwa. Rolling discs and their applications. J. of Design and Manufacturing, 2:71-82, 1992. 